\title{
Linx
}

Revue des linguistes de l'université Paris X Nanterre

38 | 1998

L'acquisition de la temporalité en situation bilingue

\section{La temporalité dans les récits sur images d'enfants bilingues franco-coréens dans leurs deux langues}

Temporality in narrative discourse of French-Korean bilingual children in their two languages

Jin-Nam Choi

\section{(2) OpenEdition}

\section{Journals}

Édition électronique

URL : http://journals.openedition.org/linx/859

DOI : $10.4000 /$ linx.859

ISSN : 2118-9692

Éditeur

Presses universitaires de Paris Nanterre

Édition imprimée

Date de publication : 1 décembre 1998

Pagination : $35-57$

ISSN : 0246-8743

Référence électronique

Jin-Nam Choi, «La temporalité dans les récits sur images d'enfants bilingues franco-coréens dans leurs deux langues », Linx [En ligne], 38 | 1998, mis en ligne le 26 juin 2012, consulté le 19 avril 2019

URL : http://journals.openedition.org/linx/859; DOI : 10.4000/linx.859

Ce document a été généré automatiquement le 19 avril 2019

Département de Sciences du langage, Université Paris Ouest 


\section{La temporalité dans les récits sur images d'enfants bilingues franco- coréens dans leurs deux langues}

Temporality in narrative discourse of French-Korean bilingual children in their two languages

Jin-Nam Choi

\section{Introduction}

1 A un stade donné de l'acquisition de la langue, l'enfant dispose d'une compétence linguistique multiple qui lui permet d'accomplir des tâches communicatives dans lesquelles il doit mettre en œuvre les moyens linguistiques propres à un type de texte donné. Le récit est ici notre objet d'étude. Les productions narratives des enfants à partir de supports d'images permettent d'analyser les processus d'élaboration d'une représentation globale de l'événement complexe et de sa mise en texte (nous distinguons entre les phases de conceptualisation et de formulation, en suivant Levelt 1989). Ces productions sont donc un objet d'étude doublement intéressant pour aborder le développement linguistique et cognitif de l'enfant.

2 A partir de cette optique théorique, le présent $\operatorname{article}^{1}$ vise à caractériser les moyens linguistiques utilisés pour marquer la temporalité et les relations temporelles dans des récits chez des enfants bilingues d'un couple de langues typologiquement très différentes : le français et le coréen, et de dégager certaines caractéristiques de la mise en œuvre des structures linguistiques du récit dans ces deux langues au cours de l'acquisition bilingue.

3 Cet article met l'accent plutôt sur quelques aspects centraux de la formulation linguistique du récit que sur ceux de la conceptualisation et la structure globale du récit. Tous ces aspects font l'objet d'une recherche plus vaste ${ }^{2}$ qui porte sur le bilinguisme 
successif enfantin afin d'observer le développement de la L1 et la L2 et de valider les notions de langues forte / langue faible à travers les indices de ces statuts différents dans la production et dans le développement linguistique et cognitif.

L'étude est centrée sur l'analyse temporelle des récits dans chaque langue :

- analyse de la macro-structure des récits, notamment la répartition des propositions entre la trame et l'arrière-plan, et la façon dont l'enfant sélectionne et met en relation les différents procès constituant un macro-événement ;

- analyse de la micro-structure pour ce qui est de la temporalité : sélection des procès, temporalité inhérente de ceux-ci; relations temporelles, marquage morphologique de la temporalité, de l'aspectualité, et connecteurs.

5 Toutes ces analyses sont une première étape de recherche sur l'acquisition des moyens linguistiques de la temporalité chez les enfants bilingues pour le couple de langue français-coréen.

\section{Profil des cinq enfants}

6 Pour cette recherche, j'ai étudié dix enfants bilingues âgés de 7 à 8 ans et demi (Choi, 1996 ; les données utilisées pour cet article concernent cinq enfants). Leur durée de séjour en France varie entre 3 ans et 7 ans - il s'agit de l'acquisition d'un bilinguisme dit 'successif' chez ces enfants'. Ceux-ci sont placés dans une situation de contact de langues très intéressante: ils parlent tous coréen en famille avec leurs parents, et vont tous à l'église pour suivre le cours biblique en coréen tous les dimanches. Les enfants 1 (Yosep) et 4 (Siwan) ont une soeur avec laquelle ils parlent coréen. C'est à l'école qu'ils pratiquent le français. Les enfants 2 (Hei-in) et 3 (Hei-jin) sont des soeurs. Elles parlent français entre elles. L'enfant 5 (Hyun-jong) est fille unique. En dehors de l'école, elle parle en coréen la plupart du temps. Les asymétries entre leurs situations linguistiques en coréen et en français font qu'ils n'ont pas tous la même langue dominante. Le profil linguistique général des cinq enfants est représenté dans le tableau suivant ${ }^{4}$ :

Tableau 1 : Profils linguistiques des enfants

\begin{tabular}{|c|c|c|c|c|c|c|}
\hline \multirow[t]{2}{*}{ Enfant } & \multicolumn{3}{|c|}{ Langue utilisée } & \multirow{2}{*}{$\begin{array}{l}\text { Séjour en Fr } \\
\text { ans ;mois }\end{array}$} & \multirow[t]{2}{*}{$L F>$ lf } & \multirow{2}{*}{\begin{tabular}{|l} 
âge \\
ans ;mois
\end{tabular}} \\
\hline & Maison & Ecole & Eglise & & & \\
\hline 1 (Yosep) & coréen & français & $\mathrm{co}\rangle \mathrm{fr}$ & $3 ; 2$ & $\operatorname{co}>\mathrm{fr}$ & $7 ; 4$ \\
\hline 2 (Hei-in) & $\mathrm{co} \approx \mathrm{fr}$ & français & co $\langle$ FR & $7 ; 3$ & FR $>c o$ & $8 ; 2$ \\
\hline $3 *($ Hei-jin $)$ & $\mathrm{co} \approx \mathrm{fr}$ & français & $\mathrm{co} \approx \mathrm{fr}$ & $7 ; 3$ & $\mathrm{fr} \approx \mathrm{co}$ & $7 ; 3$ \\
\hline 4 (Siwan) & coréen & français & $\mathrm{co}<\mathrm{fr}$ & $3 ; 4$ & $\operatorname{co}>\mathrm{fr}$ & $8 ; 2$ \\
\hline 5 (Hyun-jong) & coréen & français & co $\langle F R$ & $6 ; 5$ & $\mathrm{fr} \approx \mathrm{co}$ & 7 ; 6 \\
\hline
\end{tabular}

* $3^{*}$ : la petite soeur de Hei-in * co (coréen) *fr (français) *COfr : l'utilisation du coréen est plus forte. * FR (co : l'utilisation du français est plus forte. ${ }^{*} \mathrm{co}$ fr : I'utilisation du français et du coréen est plus ou moins équilibrée * LF langue forte * If langue faible. 


\section{Le milieu familial comme communauté bilingue chez les enfants franco-coréens}

7 Il faut remarquer que ces enfants ont des parents qui sont en France pour une période limitée avec un objectif précis, c'est-à-dire qu'ils sont soucieux de l'adaptation scolaire en France et pensent également à la réadaptation scolaire de retour en Corée. Ils espèrent que leurs enfants deviendront bilingues. Mais ces mêmes parents ont des difficultés à s'adapter à la vie française. Ils sont placés dans un environnement peu propice pour faire des progrès en français : ils ne se réunissent qu'entre eux, ils restent assez fermés vis-àvis des francophones. Les parents de Yosep par exemple sont en France pour des raisons professionnelles (le père est chercheur dans une entreprise coréenne), leurs principaux soucis vis-à-vis du français sont les problèmes scolaires de leurs enfants. Ces parents se trouvent dans une situation où les contacts et les besoins communicatifs minimaux en français leur suffisent. En conséquence, les enfants participent rarement à des situations de communication en français en dehors du contexte scolaire. Ils pratiquent tous au quotidien leur première langue. Par ailleurs, pour les grandes vacances, ils vont toujours en Corée, et commencent à oublier ce qu'ils ont appris de leur deuxième langue. La pratique de la deuxième langue est donc liée à l'école, et s'instaure éventuellement avec les frères et sœurs scolarisés (c'est le cas de Hei-in et Hei-jin). Ils ne parlent pas non plus français entre enfants quand ils se rencontrent. Les enfants récemment arrivés en France ne veulent pas s'exprimer en français avec leurs compatriotes (c'est une attitude psychologique qui se retrouve aussi chez les adultes coréens bilingues). Tous ces aspects du contexte familial semblent freiner l'accès des enfants à un bon niveau en deuxième langue, c'est donc en général un bilinguisme asymétrique qui s'instaure. Nous allons le confirmer à travers nos analyses.

\section{Méthodologie de recherche}

\section{La situation de recueil de données}

En vue de l'analyse de la temporalité dans le récit, il fallait obtenir des textes cohérents analysables et comparables. Pour ce faire, j'ai utilisé deux séquences d'images (l'histoire du chat, et l'histoire du cheval, tirées des recherches de Hickmann (1994), Hickmann et al. (1994) présentant une structure narrative cohérente à développer. Le récit est déclenché par une question du type “' 'Tu peux me raconter ce qui se passe ?' ou 'Raconte-moi cette histoire' " qui demande aux enfants de construire un récit, et implique la mise en œuvre de certains moyens linguistiques pour ce faire ${ }^{5}$.

Il est utile de noter les quelques différences existant entre ces deux histoires au niveau de la structure narrative du scénario :

a) L'histoire du cheval contient un protagoniste principal (le cheval) et deux protagonistes secondaires (la vache et l'oiseau). Au niveau de la structure temporelle, cette histoire ne présente pas de complexité: les événements se suivent de façon chronologique. Elle est composée de six images.

b) Dans l'histoire du chat, il n'y a pas de distinction nette entre protagoniste principal et protagonistes secondaires : un protagoniste (le chien) apparait un peu tardivement, un 
autre protagoniste (la maman oiseau) réapparaît après une sortie temporaire. Du point de vue de la structure temporelle, cette histoire présente plus de complexité, notamment la simultanéité entre événements, c'est-à-dire différentes actions accomplies par différents actants sur la même image. Les relations de cause et de but sont également plus centrales dans ce scénario narratif. Cette histoire est donc plus complexe que celle du cheval. Elle est composée de cinq images.

Ces différences peuvent laisser supposer que la construction de l'histoire du chat demande plus d'effort cognitif pour la constitution de la trame, et que les relations entre événements doivent être spécifiées davantage. Du point de vue linguistique, elle exige en outre une bonne maîtrise de la référence aux entités et de ses enchaînements dans l'ensemble du récit.

\section{Méthode d'enregistrement}

11 Chaque histoire est racontée par les sujets successivement dans les deux langues : en coréen et en français, à deux interlocuteurs différents. Comme les narrateurs sont des enfants bilingues qui ont une compétence narrative inégale selon les langues, j'ai demandé aux mères des enfants de susciter les récits en coréen. Les mères des enfants ont parfaitement compris l'objectif du travail et s'y sont intéressées après une discussion personnelle. J'ai laissé chaque mère faire son enregistrement à la maison en précisant les consignes suivantes :

- laisser à l'enfant quelques minutes avec les images pour lui permettre de bien comprendre le déroulement de l'histoire ;

- ne pas trop intervenir, mais aider l'enfant à se centrer au maximum sur les événements (c'est-à-dire faire raconter ce qui se passe) plutôt que de se borner à une simple description des images. Pour ce faire, la mère peut poser des questions et présenter des mots-clés.

Pour l'enregistrement des récits en français, j'ai attendu la réunion des mères et des enfants, sous forme d'une visite amicale entre familles, chez l'enfant 4. Les enregistrements ont été faits par moi-même et se sont passés dans une pièce à l'écart. Les enfants ont raconté l'un après l'autre. Vu le milieu linguistique et familial, les enfants, qui parlent entre eux en français ${ }^{6}$, étaient très contents de pouvoir rencontrer en semaine leurs amis compatriotes. Cette situation a favorisé la narration en français. L'enregistrement a duré à peu près 5 minutes par récit.

Tableau 2 : La collecte des données

\begin{tabular}{|l|l|l|l|l|}
\hline & \multicolumn{2}{|l|}{$\begin{array}{l}\text { L'ordre de l'enregistrement } \\
\text { (récit en fr, récit en co) }\end{array}$} & \multicolumn{2}{l|}{ Type d'enregistrement } \\
\hline Enfant & premièrement & deuxièmement & D* & SI ** \\
\hline $\mathbf{1}$ & récit en coréen & récit en français & & SI \\
\hline $\mathbf{2}$ & récit en coréen & récit en français & D & \\
\hline $\mathbf{3}$ & récit en coréen & récit en français & D & \\
\hline
\end{tabular}




\begin{tabular}{|l|l|l|l|l|}
\hline 4 & récit en coréen & récit en français & & SI \\
\hline 5 & récit en coréen & récit en français & D & \\
\hline
\end{tabular}

*à distance ** succession immédiate

\section{Analyse de corpus}

Pour pouvoir mettre en évidence les différents paramètres susceptibles d'influer sur les données, nous présentons les analyses temporelles sous forme de tableaux. Mais d'abord, nous donnons un exemple d'analyse de la temporalité dans un passage de récit dans chaque langue. L'analyse des corpus est présentée en trois colonnes: la première correspond aux énoncés segmentés en propositions ${ }^{7}$, la deuxième colonne du tableau présente l'information temporelle reconstruite par interprétation pour chaque proposition, dont les caractéristiques temporelles internes aux procès (états ou événements) concernés, et la troisième colonne spécifie les moyens linguistiques utilisés pour construire l'information temporelle. On y trouve trois sous-colonnes: celle de l'aspect, celle de la morphologie verbale et celle des connecteurs. Pour le récit en coréen, le corpus est transcrit en translittération, accompagné d'une glose morphémique et d'une traduction en français.

Le modèle d'analyse est basé sur la grille d'analyse de la temporalité dans les textes d'apprenants de Noyau $(1990,1991)$ et le cadre de la temporalité de Klein $(1994,1995)$ qui sont à base conceptuelle, ainsi que sur le modèle de la Quaestio (Klein et von Stutterheim 1987, 1991), pour ce qui est du mouvement référentiel dans les textes. La Quaestio permet de décrire les structures informationnelles, c'est-à-dire d'identifier la distribution des informations dans le discours et la manière dont l'information se déroule d'un énoncé à l'autre à travers le texte dans les différents domaines référentiels, temps, entité, espace, modalité, procès. Ces différents domaines référentiels peuvent varier selon le type de discours, c'est-à-dire un domaine référentiel peut être plus privilégié dans un discours que dans d'autre: récit (temps, procès), description (espace et entité). Dans cet article, nous nous limitons à décrire la structuration globale du récit: trame (la structure principale) et l'arrière-plan (la structure secondaire) les relations temporelles entre propositions, et la morphologie tempero-aspectuelle.

Le modèle conceptuel de la temporalité que nous avons adopté (Noyau 1991, Klein 1994) distingue trois types d'intervalles temporels : le moment de la parole (MP), le moment de la situation (MS), qui est l'intervalle temporel occupé par le procès, et le moment en question (MQ), qui est l'intervalle temporel sur lequel porte l'assertion. Les relations temporelles de base comprennent les relations d'ordre : relation avant ou d'antériorité $(\mathrm{AV})$, et relation après ou de postériorité (APR), ainsi que des relations topologiques: concomitance (CONT), adjacence (+/-ADJ) et proximité (+/-PROX). Les relations entre les intervalles MQ et MP au niveau de chaque proposition décrivent la temporalité stricte, celles entre les intervalles temporels MQ et MS décrivent l'aspectualité8. Klein (1994) propose de distinguer seulement trois grands types de procès, procès à 0-état (propriété constitutive d'une entité, pour laquelle il n'y a pas de contraste entre MQs), procès à 1état (état contingent d'une entité, ou activité homogène, le MQ est alors en contraste avec 
d'autres MQs), et procès à 2-états (passage d'un état à un autre état, où on a MQ1 associé à un état initial, et MQ2 associé à un état final).

Par ailleurs, le modèle de la quaestio (Klein \& Stutterheim 1991) définit des contraintes de la référenciation qui portent sur les propositions constituant la trame d'un texte structuré. Pour ce qui est du récit, ces contraintes sur les propositions de la trame comprennent entre autres les suivantes, centrales pour notre propos :

- les prédicats doivent être des procès singuliers occupant un intervalle temporel défini ;

- il y a une relation de consécutivité entre les intervalles temporels (MQs) associés aux propositions successives de la trame (principe de l'ordre naturel ou chronologique).

Tableau 3 : Analyse d'un récit en français

Enfant 1 Yosep. Histoire du chat en français

\begin{tabular}{|c|c|c|c|c|}
\hline Segments & Traits temporels & Moyens ling & uistiques & \\
\hline${ }^{*} \mathrm{~T}$ :trame & $\begin{array}{l}\text { a) relations } \\
\text { temporelles } \\
\text { b) temporalité } \\
\text { inhérente } \\
\text { des procès }\end{array}$ & aspect & $\begin{array}{l}\text { Morphologie } \\
\text { verbale } \\
\text { 'lexique des } \\
\text { procès' }\end{array}$ & connet \\
\hline $\begin{array}{l}\text { 1. il [iave] un oiseau et les petits } \\
\text { oiseaux }\end{array}$ & $\begin{array}{l}\text { MS1 } \\
\text { MQ1 =MS1 } \\
1^{\mathrm{E}}\end{array}$ & imperfectif & $\begin{array}{l}\text { IMP } \\
\text { 'il y a' }\end{array}$ & \\
\hline $\begin{array}{l}\text { T2. Et l'oiseau est parti / pour } \\
\text { manger } \\
+ \text { /à apporter à manger }\end{array}$ & $\begin{array}{l}\text { MS2 CONT MS1 } \\
\text { MQ1INCL MS2 } \\
2^{\mathrm{E}}\end{array}$ & perfectif & $\begin{array}{l}\mathrm{PC} \\
\text { 'partir à } \\
\text { apporter' }\end{array}$ & et \\
\hline 3. et il [iave] un chat qui e/e là & $\begin{array}{l}\text { MS3 CONT MS1-2 } \\
\text { MQ1 IN MS3 } \\
1^{\mathrm{E}}\end{array}$ & imperfectif & $\begin{array}{l}\text { IMP + PREST } \\
\text { 'il y a' + ' être' }\end{array}$ & et \\
\hline $\begin{array}{l}\text { T4. Alors il a vu les petits } \\
\text { oiseaux }\end{array}$ & $\begin{array}{l}\text { MS4 APR MS2 } \\
\text { MQ3 INCL MS4 } \\
1^{\mathrm{E}}\end{array}$ & perfectif & $\begin{array}{l}\mathrm{PC} \\
\text { 'voir' }\end{array}$ & alors \\
\hline $\begin{array}{l}\text { 5. après il [vule] manger les } \\
\text { oiseaux }\end{array}$ & $\begin{array}{l}\text { MS5 CONT MS4 } \\
\text { MQ1 IN MS5 } \\
1^{\mathrm{E}}\end{array}$ & imperfectif & $\begin{array}{l}\text { IMP } \\
\text { 'vouloir manger' }\end{array}$ & après \\
\hline T6. Alors il a [mõte] dans l'arbre & $\begin{array}{l}\text { MS6 APR MS4 } \\
\text { MQ1 INCL MS6 } \\
2^{\mathrm{E}}\end{array}$ & perfectif & $\begin{array}{l}\text { PC } \\
\text { 'monter' }\end{array}$ & alors \\
\hline
\end{tabular}




\begin{tabular}{|l|l|l|l|l|}
\hline $\begin{array}{l}\text { T7. Mais il y a un chien qui } \\
\text { [evənu] }\end{array}$ & $\begin{array}{l}\text { MS7 APR MS6 } \\
\text { MQ7 INCL MS7 } \\
2^{\text {E }}\end{array}$ & perfectif & $\begin{array}{l}\text { PC } \\
\text { 'venir' }\end{array}$ & mais \\
\hline $\begin{array}{l}\text { T8. Après le chien il [afepamõte] } \\
\text { le chat }\end{array}$ & $\begin{array}{l}\text { MS8 APR MS7 } \\
\text { MQ7INCL MS8 } \\
2^{\text {E }}\end{array}$ & perfectif & $\begin{array}{l}\text { PC } \\
\text { 'faire } \\
\text { monter' }\end{array}$ & pas \\
\hline $\begin{array}{l}\text { T9. Après le/l'oiseau il [aaporte] } \\
\text { à manger. }\end{array}$ & $\begin{array}{l}\text { MS9 APR MS8 } \\
\text { MQ7 INCL MS9 } \\
2^{\text {E }}\end{array}$ & perfectif & PC & après \\
& & 'apporter' & \\
\hline
\end{tabular}

${ }^{*} \mathrm{OE}$ : verbe à 0 état * $1 \mathrm{E}:$ verbe à 1 état *2E : verbe à 2 état * ${ }^{*} \mathrm{O}=$ connecteurs

${ }^{*}$ CONNECT $=\mathbf{~ C O N N E C T E U R S ~}{ }^{*} \mathbf{M S}:$ moment de la situation ${ }^{*} \mathbf{M Q}:$ moment en question ${ }^{*} \mathbf{M P}$ moment de la parole

*PC : passé composé *IMP : imparfait *PP : participe passé

\section{Récit en coréen}

17 Pour la lisibilité du corpus coréen, nous allons montrer comment chaque segment est glosé morphémiquement. Par exemple:

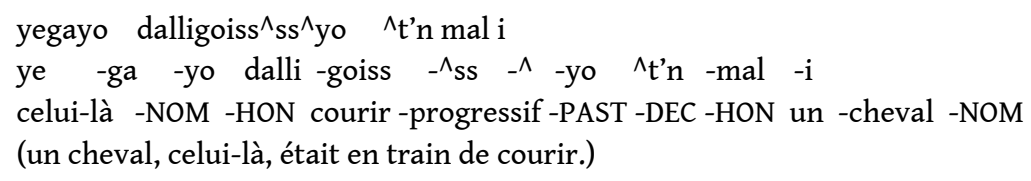

Chaque segment transcrit est accompagné d'une glose morphémique en français juste audessous du morphème correspondant en coréen. La traduction globale en français est donnée entre parenthèses.

19 Pour fournir une idée du système temporo-aspectuel coréen, je présente ci-dessous un tableau des morphèmes temporels avec les valeurs aspectuelles qui leur sont associées, réécrits d'après la grille d'analyse, ainsi que les structures périphrastiques d'aspect. Mentionnons les schèmes d'aspect périphrastiques pour quatre notions aspectuelles: aspect progressif '-goiss' (être en train de), aspect continuatif '-n(n jugi' (dans l'activité de), aspect itératif '-gonha', aspect inchoatif '-mag-V-hagi sijagha' (juste-V commencer à).

Tableau 4 : les morphèmes temporels et les valeurs aspectuelles qui leur sont associés selon les classes de procès :

\begin{tabular}{|l|l|l|}
\hline & schèmes & valeurs \\
\hline $\begin{array}{l}\text { présent } \\
\text { (VDPREST })\end{array}$ & $\begin{array}{l}\mathrm{V}+\varnothing-\mathrm{da} \\
\text { (nÎn) }\end{array}$ & $\begin{array}{l}\text { MQ CONT MP } \\
\text { MS INCL MQ }\end{array}$ \\
\hline $\begin{array}{l}\text { passé1 } \\
(\mathrm{P} 1+\mathrm{VD})\end{array}$ & $\begin{array}{l}\text { V+ ass-da } \\
(\wedge \mathrm{ss})\end{array}$ & $\begin{array}{l}\text { MQ AV MP } \\
\text { MS INCL MQ }\end{array}$ \\
\hline
\end{tabular}




\begin{tabular}{|l|c|l|}
\hline $\begin{array}{l}\text { passé1+passé1 } \\
(\mathrm{P} 1+\mathrm{P} 1)\end{array}$ & $\begin{array}{l}\mathrm{V}+\text { ass } \\
\left({ }^{\wedge} \mathrm{ss} \mathrm{s}^{\wedge} \mathrm{ss}\right)\end{array}$ & $\begin{array}{l}\text { MQ AV MP } \\
\text { MS INCL MQ }\end{array}$ \\
\hline $\begin{array}{l}\text { passé2 +passé1 } \\
(\mathrm{P} 2+\mathrm{P} 1)\end{array}$ & $\begin{array}{c}\mathrm{V}+\mathrm{d}^{\wedge}+\text { 'ass'-da } \\
\left({ }^{\wedge} \mathrm{ss}\right)\end{array}$ & $\begin{array}{l}\text { MQ AV MP } \\
\text { MS INCLMQ }\end{array}$ \\
\hline $\begin{array}{l}\text { futur } \\
\text { (FUTUR) }\end{array}$ & $\begin{array}{l}\mathrm{V} \text { gess-da } \\
\text { MQ APR MP } \\
\text { MS CONT MQ }\end{array}$ \\
\hline
\end{tabular}

*( ) : variante

Tableau 5 : L 'enfant 1. Yosep. Histoire du cheval en coréen

\begin{tabular}{|c|c|c|c|c|}
\hline Segments & Traits temporels & Moyens ling & uistiques & \\
\hline${ }^{*} \mathrm{~T}$ :trame & $\begin{array}{l}\text { a) relations temporelles } \\
\text { b) temporalité inhérente } \\
\text { des procès }\end{array}$ & aspect & $\begin{array}{l}\text { Morphologie } \\
\text { verbale } \\
\text { 'lexique des } \\
\text { procès' }\end{array}$ & cco \\
\hline $\begin{array}{l}\text { ye -ga -yo dalli -goiss - } \\
\wedge_{\text {ss }} \\
\text { celui-là -NOM -HON courir - } \\
\text { gérondif -PAST } \\
-\wedge \text {-yo } \wedge^{\prime} \text { 'n -mal -i } \\
\text {-DEC-HON un -cheval -NOM } \\
\text { (un cheval, celui-là, était en } \\
\text { train de courir.) }\end{array}$ & $\begin{array}{l}\text { MS1 } \\
\text { MQ1 = MS1 } \\
\text { MQ1 IN MS1 } \\
1^{\mathrm{E}}\end{array}$ & $\begin{array}{l}\text { imperfectif } \\
\text { progressif }\end{array}$ & $\begin{array}{l}\mathrm{P} 1+\mathrm{VD} \\
\text { " goiss" } \\
\text { 'courir' }\end{array}$ & \\
\hline $\begin{array}{l}\text { T2. Gîlegagigu-yo ye -ga - } \\
\text { yo mal -i } \\
\text { et après -HON celui-là-NOM - } \\
\text { HON cheval-NOM } \\
\text {-yo so -lîl bo -ass -g^dîn } \\
\text {-yo } \\
\text {-HON vache -ACC voir -PAST - } \\
\text { DEC -HON } \\
\text { (et après ce cheval-là a vu la } \\
\text { vache) }\end{array}$ & $\begin{array}{l}\text { MS2 CONT MS1 } \\
\text { MQ1 INCL MS2 } \\
2^{\mathrm{E}}\end{array}$ & perfectif & $\begin{array}{l}\text { P1+VD } \\
\text { 'voir' }\end{array}$ & $\begin{array}{l}\text { et } \\
\text { après }\end{array}$ \\
\hline
\end{tabular}




\begin{tabular}{|c|c|c|c|c|}
\hline 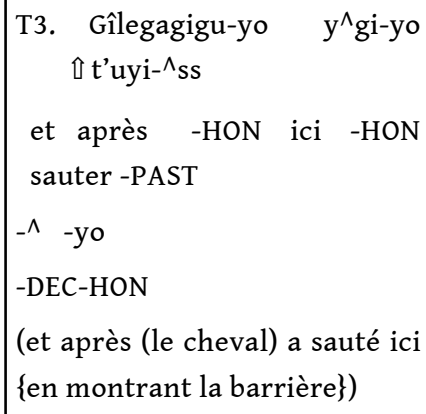 & $\begin{array}{l}\text { MS3 APR MS2 } \\
\text { MQ1 INCL MS3 } \\
2^{\mathrm{E}}\end{array}$ & perfectif & $\begin{array}{l}\mathrm{P} 1+\mathrm{VD} \\
\text { 'sauter' }\end{array}$ & $\begin{array}{l}\text { et } \\
\text { après }\end{array}$ \\
\hline 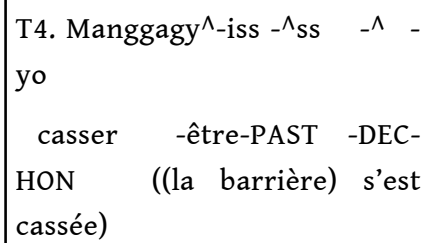 & $\begin{array}{l}\text { MS4 APR MS3 } \\
\text { MQ1 INCL MS4 } \\
2^{\mathrm{E}}\end{array}$ & perfectif & $\begin{array}{l}\mathrm{P} 1+\mathrm{VD} \\
\text { 'se casser' }\end{array}$ & \\
\hline $\begin{array}{l}\text { 5. gîlegagigu-yo } \Uparrow \text { t'uyi }-\wedge \text { Ss } \\
-\wedge \text {-yo } \\
\text { et après -HON sauter -PAST } \\
\text {-DEC-HON } \\
\text { (et après (le cheval) a sauté.) }\end{array}$ & $\begin{array}{l}\text { MS5 = MS3 } \\
\text { MQ1 INCL MS5 } \\
2^{\mathrm{E}}\end{array}$ & perfectif & $\begin{array}{l}\mathrm{P} 1+\mathrm{VD} \\
\text { 'sauter' } \\
\text { reprise de MS3 }\end{array}$ & $\begin{array}{l}\text { et } \\
\text { après }\end{array}$ \\
\hline $\begin{array}{l}\text { 6a. gîlegagigu-yo t'yi -dag } \\
-\wedge \text {-yo } \\
\text { et après -HON sauter -en } \\
\text { faisant -DEC-HON } \\
\text { (et après en sautant) }\end{array}$ & $\begin{array}{l}\text { MS6a CONT MS3-5 } \\
\text { MQ6a }= \\
2^{\text {E }}\end{array}$ & & $\begin{array}{l}\text { gérondif } \\
\text { 'sauter' }\end{array}$ & \\
\hline $\begin{array}{l}\text { T6b. euh } \operatorname{dac}(\wedge)-{ }^{\wedge} \text { ss } \\
\text { euh se blesser -PAST } \\
\text { (et après le cheval s'est } \\
\text { blessé.) }\end{array}$ & $\begin{array}{l}\text { MS6b CONT MS6a } \\
\text { MQ6a INCL MS6b } \\
2^{\mathrm{E}}\end{array}$ & perfectif & $\begin{array}{l}\mathrm{P} 1+\mathrm{VD} \\
\text { 'se blesser' }\end{array}$ & $\begin{array}{l}\text { et } \\
\text { après }\end{array}$ \\
\hline $\begin{array}{l}\text { T7. Gîl^nik'a so -ga } \\
\text { bwagagi -go -yo } \\
\text { alors vache -NOM voir - } \\
\text { CONJ-HON (alors la vache a } \\
\text { vu) }\end{array}$ & $\begin{array}{l}\text { MS7 APR MS5 } \\
\text { MQ1 INCL MS7 } \\
2^{\mathrm{E}}\end{array}$ & perfectif & $\begin{array}{l}\mathrm{P} 1+\mathrm{VD} \\
\text { 'voir' }\end{array}$ & alors \\
\hline $\begin{array}{l}\text { T8. Ganhow }{ }^{\wedge} \mathrm{n} / \text { ganhow }^{\wedge} \mathrm{n} \\
\text { gabang -îl } \\
\text { infirmière / infirmière sac } \\
\text {-ACC } \\
\text { gaj^}{ }^{\wedge} \mathrm{wa} \text { / gagigu-yo } \\
\text { apporter/ avec -HON } \\
\text { (et (la vache) a apporté la } \\
\text { boîte à l'infirmière) }\end{array}$ & $\begin{array}{l}\text { MS8 APR MS7 } \\
\text { MQ1 INCL MS8 } \\
2^{\mathrm{E}}\end{array}$ & perfectif & $\begin{array}{l}\mathrm{P} 1+\mathrm{VD} \\
\text { 'apporter' }\end{array}$ & et \\
\hline
\end{tabular}




\begin{tabular}{|c|c|c|c|}
\hline $\begin{array}{l}\text { T9. Dali -lîl goc }{ }^{\wedge} \mathrm{ju}-{ }^{\wedge} \mathrm{ss}-\wedge \\
\text {-yo. } \\
\text { Patte -ACC faire guérir - } \\
\text { PAST-DEC-HON } \\
\text { ((elle) a soigné la patte du } \\
\text { cheval.) }\end{array}$ & $\begin{array}{l}\text { MS9 APR MS8 } \\
\text { MQ1 INCL MS9 } \\
1^{\mathrm{E}}\end{array}$ & perfectif & $\begin{array}{l}\mathrm{P} 1+\mathrm{VD} \\
\text { 'soigner' }\end{array}$ \\
\hline
\end{tabular}

\section{Procédure d'analyse}

21 A partir du cadre théorique adopté, que nous avons présenté ci-dessus (cf. Klein 1994, Klein \& Stutterheim 1991, Noyau 1991), pour procéder à l'analyse temporelle des récits dans les deux langues, nous avons d'abord effectué l'analyse de chaque récit au niveau global conditionné par la quaestio sous-jacente. Cela a donné la distribution de l'information dans les textes entre les propositions de la trame (celles qui répondent directement à la quaestio, c'est-à-dire les propositions d'événements singuliers ordonnées chronologiquement) et celles de l'arrière-plan (qui ne répondent pas directement à la quaestio). Les propositions de la trame sont mises en gras dans l'analyse. Ensuite, nous avons analysé les marquages linguistiques de la temporalité utilisés dans la trame et l'arrière-plan dans les deux langues. Ce modèle détermine donc la façon dont l'information est sélectionnée en vue de la construction globale du récit: trame et arrière-plan, et décrit les caractéristiques de différents moyens linguistiques qui sont propres à un type de discours comme récit.

\section{Résultats de l'analyse}

Nous présentons ici les résultats concernant a) la distribution des moyens linguistiques temporels dans la trame et dans l'arrière plan, b) les différences existant entre les productions dans ces deux langues (la langue forte et la langue faible) chez ces enfants bilingues. Ils seront répartis en quatre tableaux pour chaque langue: le français et le coréen. A travers ces résultats, nous allons faires quelques remarques sur les formulations linguistiques du récit.

Les tableaux 6, 7 et 10, 11 rendent compte des moyens linguistiques utilisés dans les propositions de la trame : les tableaux 6, 7 concernent les propositions de la trame dans les récits en français et les tableaux 10 et 11 concernent les propositions de la trame dans les récits en coréen. Les tableaux 8, 9 et 12, 13 regroupent des moyens linguistiques utilisés dans les propositions de l'arrière-plan, pour les récits en français (8 et 9) et pour les récits en coréen (12 et 13).

Dans les tableaux, quatre colonnes indiquent : la première, le numéro d'ordre de chaque enfant, la deuxième, le nombre total des propositions du récit (Tprop), la troisième, le nombre de propositions appartenant à l'un des deux plans de discours traité, i.e., trame (Tra) ou arrière-plan (AP), et la quatrième le pourcentage des propositions qui sont dans la trame ( \%Tra) ou dans l'arrière-plan (\%AP). 
Tableau 6 : Distribution des marques morphologiques dans les propositions de la TRAME des récits en français ${ }^{9}$

\begin{tabular}{|l|l|l|l|l|l|l|l|l|l|l|l|l|l|}
\hline Enfant & TProp & Tra & $\begin{array}{l}\% \\
\text { Tra }\end{array}$ & $\begin{array}{l}\text { PQP } \\
\mathrm{n}^{\circ}\end{array}$ & $\begin{array}{l}\text { PS } \\
\mathrm{n}^{\circ}\end{array}$ & $\begin{array}{l}\% \\
\mathrm{n}^{\circ}\end{array}$ & $\begin{array}{l}\text { PC } \\
\mathrm{n}^{\circ}\end{array}$ & $\begin{array}{l}\text { IMP } \\
\mathrm{n}^{\circ}\end{array}$ & $\begin{array}{l}\text { PREST } \\
\%\end{array}$ \\
\hline 2 & 19 & 12 & 63.1 & & & & & 10 & 83.3 & & & 1 & 8.3 \\
\hline 3 & 24 & 11 & 45.8 & 2 & 18.0 & 9 & 81.1 & & & & & & \\
\hline 4 & 24 & 11 & 45.8 & & & 5 & 45.4 & 2 & 18.1 & $1 ?$ & 9.0 & 3 & 27.2 \\
\hline 5 & 40 & 18 & 45.0 & 1 & 5.5 & $1 ?$ & 5.5 & 7 & 38.8 & 1 & 5.5 & 7 & 38.8 \\
\hline Total & 147 & 69 & 46.9 & 3 & 4.0 & 15 & 21.7 & 23 & 33.0 & 2 & 2.8 & 24 & 34.7 \\
\hline
\end{tabular}

*TProp : Nombre total de propositions dans le récit *Ptra : Nombre de propositions de la trame * \% PTra : pourcentage des propositions de la trame. ${ }^{*} P Q P$ : plus-que-parfait *PS : passé simple *PC : passé composé

*IMP : imparfait *PREST : présent * $n^{\circ}$ : nombre d'occurrences

Tableau 7 : Distribution des adverbes temporels et des connecteurs dans les propositions de la TRAME des récits en français

\begin{tabular}{|c|c|c|c|c|c|c|c|c|}
\hline & & & Conn & eurs te & orels & & connecteurs & adverbes temporels \\
\hline Enfant & Tprop & Tra & $\begin{array}{l}\text { (et) } \\
\text { puis }\end{array}$ & $\begin{array}{l}\text { (et) } \\
\text { après }\end{array}$ & (et)alors & et & & \\
\hline 1 & 19 & 12 & & 7 & 2 & 2 & & \\
\hline 2 & 40 & 17 & & & & 4 & $\begin{array}{l}\text { mais (4), donc } \\
(1)\end{array}$ & \\
\hline 3 & 24 & 11 & & & & & & $\begin{array}{l}\text { pd ce to-là(1) soudain } \\
\text { (1) }\end{array}$ \\
\hline 4 & 24 & 11 & 1 & & & 4 & mais (2) & \\
\hline 5 & 40 & 18 & & & 1 & 10 & & \\
\hline Total & 147 & 69 & 1 & 7 & 3 & 20 & $\begin{array}{l}\text { mais (6), donc } \\
\text { (1) }\end{array}$ & $\begin{array}{l}\text { pd ce to-là(1) soudain } \\
\text { (1) }\end{array}$ \\
\hline
\end{tabular}

Les tableaux 6 et 7 donnent la répartition des moyens linguistiques utilisés par nos enfants bilingues dans la trame du récit. Le nombre total des propositions pour les 5 récits est 147 propositions (dorénavant prop) en moyenne de 29.4 prop par récit. Le 
pourcentage de proposition de la trame, $40 \%$, est inférieur par rapport aux indicateurs élaborés pour les enfants francophones du même tranche d'âge $(65 \%)^{10}$.

Les enfants utilisent dans la trame la morphologie verbale du passé composé ( $\mathrm{PC}, 23$ prop au total, soit $33 \%$ ) ou du passé simple (PS, 15 prop. au total, soit $21.7 \%$ ), les propositions à ces deux temps du passé ( 38 prop.) constituant $54.7 \%$ de l'ensemble des occurrences verbales dans les propositions (69 prop.) de la trame. On y trouve aussi des occurrences du présent (dans le cas d'un récit raconté au présent narratif : celui de l'enfant 2 Hei-in). Dans le récit de Hei-in, la trame n'est marquée que par les traits inhérents à la sémantique des verbes : les prédicats comprennent des verbes téliques représentant des procès à changement d'état 'arriver', 'grimper', 'donner à manger', par exemple :

\begin{tabular}{|l|}
\hline T9a. $₫$ donc le chat grimpe à l'arbre \\
\hline pour les atteindre \\
\hline T10. mais le chien arrive \\
\hline T11a. le chien /(11a')+ \\
\hline 11a'. qui n'aime pas le chat \\
\hline saisit par la queue \\
\hline T12. et essaie de la faire tomber \\
\hline
\end{tabular}

Si on regarde les quelques autres formes verbales dans la trame, le plus-que-parfait est utilisé avec une valeur perfective et non accomplie :

Enf5 $\mathrm{H}^{11}$ : T3. pendant ce temps-là + un chat [avevy] les petits oiseaux

Et l'imparfait est utilisé pour marquer une action en cours à un moment particulier dans le récit :

\begin{tabular}{|l|}
\hline Enf5H1 : T7. [idi] \\
\hline 7-1. tiens je vais grimper sur l'arbre \\
\hline T8. et il (ete) en train de grimper sur l'arbre. \\
\hline
\end{tabular}

30 Ici, l'imparfait est utilisé avec 'être en train de', qui est la forme périphrastique du progressif. Dans ce cas, ce n'est pas la morphologie verbale qui nous permet de dire que MQ8 APR MS7, mais c'est une inférence pragmatique par la relation entre les deux événements qui permet de le déduire.

31 Pour ce qui est des connecteurs introduisant les propositions de la trame, on peut remarquer l'utilisation fréquente du connecteur 'et '( 20 occurrences au total) quelles que 
soient les formes verbales. Ainsi que le connecteur temporel '(et) après' avec le PC, pour marquer explicitement la consécutivité entre les événements successifs de la trame du récit.

Des expressions temporelles comme 'soudain' et 'pendant ce temps-là' qui permet de construire la relation de concomitance sont utilisées. Ces expressions servent à spécifier l'intervalle temporel associé au procès :

Enf3 H1 :3) pendant ce temps-là + un chat [avevy] les petits oiseaux.

Enf3 H2 :T2. soudain il [sareta]

Tableau 8: Distribution des marques morphologiques dans les propositions de l'ARRIERE-PLAN des récits en français

\begin{tabular}{|c|c|c|c|c|c|c|c|c|c|c|c|c|c|c|c|c|c|}
\hline Enfant & Tprop & AP & $\begin{array}{l}\% \\
\mathrm{AP}\end{array}$ & $\begin{array}{l}\text { PREST } \\
\mathrm{n}^{\circ}\end{array}$ & $\%$ & $\begin{array}{l}\text { PC } \\
\text { n } \\
\text { 。 }\end{array}$ & $\%$ & $\begin{array}{l}\text { IMP } \\
n^{\circ}\end{array}$ & $\%$ & $\begin{array}{l}P Q P \\
n^{\circ}\end{array}$ & $\%$ & $\begin{array}{l}\text { Futur } \\
\mathrm{n}^{\circ}\end{array}$ & $\%$ & $\begin{array}{l}\text { CON } \\
n^{\circ}\end{array}$ & $\%$ & $\begin{array}{l}\mathrm{G} \\
\mathrm{n} \\
0\end{array}$ & $\%$ \\
\hline 1 & 19 & 7 & 36.8 & & & 1 & 14.2 & 4 & 57.1 & & & 1 & 14.2 & & & 1 & 14.2 \\
\hline 2 & 40 & 23 & 57.5 & 19 & 82.6 & 1 & 4.3 & & & & & 1 & 4.3 & 1 & 4.3 & 1 & 7.6 \\
\hline 3 & 24 & 13 & 54.1 & 5 & 38.4 & & & 6 & 46.1 & 1 & 7.6 & & & 1 & 7.6 & & \\
\hline 4 & 24 & 13 & 54.1 & 4 & 30.7 & & & 5 & 38.4 & & & 3 & 23.0 & & & & \\
\hline 5 & 40 & 22 & 55.0 & & & 2 & 9.0 & 9 & 40.9 & 1 & 4.5 & 7 & 31.8 & & & 2 & 9.0 \\
\hline Total & 147 & 78 & 53.0 & 28 & 35.8 & 4 & 5.1 & 24 & 30.7 & 2 & 2.5 & 12 & 15.3 & 2 & 2.5 & 4 & 7.1 \\
\hline
\end{tabular}

${ }^{*} \mathrm{G}$ : gérondif *CON : conditionnel

Tableau 9: Distribution des adverbes temporels et des connecteurs dans les propositions de I'ARRIERE-PLAN des récits en français

\begin{tabular}{|c|c|c|c|c|c|c|c|}
\hline & & & Connecte & ur tempor & & connecteurs & adverbes temporels \\
\hline Enfant & Tprop & AP & (et) puis & (et) alors & et & & \\
\hline 1 & 19 & 7 & & & 1 & mais & \\
\hline 2 & 40 & 23 & & & 1 & mais & toujours, cette fois \\
\hline 3 & 24 & 13 & & & 1 & & quand \\
\hline 4 & 24 & 13 & 1 & & 2 & mais(2) & \\
\hline 5 & 40 & 22 & & 1 & 2 & & tout \\
\hline
\end{tabular}




\begin{tabular}{|l|l|l|l|l|l|l|l|}
\hline Total & 147 & $\mathbf{7 8}$ & 1 & 1 & 7 & mais(4) & tjs, cette fois, qd, tout \\
\hline
\end{tabular}

Les tableaux 8 et 9 donnent la répartition des marques linguistiques utilisées dans les propositions d'arrière-plan dans les récits en français. Nous trouvons le plus souvent une relation de simultanéité dans l'AP. C'est souvent le cas d'énoncés qui ne font pas référence à des événements, mais à des états. Il s'agit donc de différents états simultanés d'un actant du récit ou deux états de deux actants différents mais dans le même espace temporel. On l'observe le plus souvent chez l'enfant 2 (Hei-in) qui a une compétence la plus forte en français.

\begin{tabular}{|l|}
\hline Enf2 $\mathrm{H} 2: 2$. il y a une vache \\
\hline 3.la vache est en train de discuter avec un oiseau \\
\hline 4.le cheval les regarde avec des yeux tout ronds \\
\hline 5.il est vraiment très étonné \\
\hline
\end{tabular}

On remarque d'abord que les formes verbales utilisées pour l'AP sont plus variées que celles pour la $\mathrm{T}$. Les enfants recourent en grande partie aux schèmes morphologiques de l'imparfait ou du présent. Ces schèmes morphologiques se manifestent notamment avec les constructions de focalisation comme 'Il y a ( iave) X qui V' pour introduire une nouvelle entité dans le récit :

\begin{tabular}{|l|}
\hline Enf.H1 :2) Il [iave] un oiseau et les petits oiseaux. \\
\hline 3a) et il [iave] un chat \\
\hline qui [ete] là \\
\hline 7a) mais il y a un chien \\
\hline
\end{tabular}

Relevons aussi les corrélations des connecteurs avec la relation temporelle de concomitance: les enfants utilisent les connecteurs 'et' ou 'mais' pour une fonction additive : 'et', ou de contraste: 'mais' entre des propositions A et B. Ces deux paires de propositions donnent une description d'événements concomitants, où $\mathrm{A}$ fait $\mathrm{X}$ et $\mathrm{A}$ (ou $\mathrm{B}$ ) fait $\mathrm{Y}$, ou bien $\mathrm{A}$ fait $\mathrm{X}$ mais $\mathrm{B}$ fait $\mathrm{Y}$.

\begin{tabular}{|l|l|}
\hline Enf2 H1 : & 3) la mère va chercher de quoi manger \\
\hline & 4) mais un chat arrive \\
\hline Enf5 H1 : & 10) et il [etearive] au nid \\
\hline & 11a) mais le chat il [save] pas \\
\hline
\end{tabular}


On constate également que mais est associé à l'introduction d'une nouvelle entité thème en topique, qui déclenche un nouvel épisode du récit. Ce phénomène est très fréquent chez Hei-in (cf. 4, 10) :

\begin{tabular}{|l|}
\hline Enf2 $\mathrm{H} 1: 3$ ) la mère va chercher de quoi manger \\
\hline 4) mais un chat arrive \\
\hline 7a) le chat attend \\
\hline 7b) qu'un des oisillons tombe ++ \\
\hline 7c) et s'envole \\
\hline 8) mais aucun d'eux ne tombe \\
\hline 9) donc le chat grimpe à l'arbre \\
\hline 10) mais le chien arrive \\
\hline
\end{tabular}

Les schèmes morphologiques du plus-que-parfait et du passé composé sont également associés à la relation temporelle de concomitance. Mais ces formes verbales accompagnent le pronom anaphorique 'ça' référant à la situation précédente.

Enf1H2 :5) il + vache + il [arəgarde] ça

La forme verbale 'aller $+V$ infinitif' (aspect prospectif MQ AV MS) est à valeur d'aspect prospectif( 8 prop., soit $10.1 \%)$. Cette forme verbale se manifeste également dans le discours rapporté : c'est le cas pour l'enfant 5 ( 6 prop., $26 \%$ du total) qui l'utilise le plus fréquemment. Ce sont alors des projets d'actions formulés dans le discours rapporté, qui sont ensuite repris dans la trame comme actions réalisées :

\begin{tabular}{|l|}
\hline E5H2 : 4) il dit \\
\hline 4-1) et ben moi je [səre] toute seule \\
\hline 4-2) alors je vais sauter de la barrière \\
\hline 4-3) et je vais jouer avec la vache \\
\hline 5) et alors le cheval [asote] euh sur la barrière \\
\hline
\end{tabular}


Tableau 10 : Distribution des marques morphologiques entre les propositions de la TRAME des récits en coréen

\begin{tabular}{|c|c|c|c|c|c|c|c|c|c|c|c|c|c|c|c|c|c|}
\hline & & & & & $\begin{array}{l}\text { PREST } \\
+\mathrm{V} 1 \mathrm{E}\end{array}$ & & $\begin{array}{l}\text { PREST } \\
+\mathrm{V} 1 \mathrm{E}+ \\
\text { «goiss }\end{array}$ & & $\begin{array}{l}\text { PREST } \\
+\mathrm{V} 2 \mathrm{E} »\end{array}$ & & $\begin{array}{l}\text { PREST } \\
+\mathrm{V} 2 \mathrm{E} \\
\text { «goiss» }\end{array}$ & & $\begin{array}{l}\text { P1 } \\
+\mathrm{V} 1 \\
\mathrm{E}\end{array}$ & & $\begin{array}{l}\mathrm{P} 1 \\
+\mathrm{V} 2 \mathrm{E}\end{array}$ & & $\begin{array}{l}\mathrm{P} 1 \\
+\mathrm{V} 2 \mathrm{E} \\
\end{array}$ \\
\hline Enfant & Tprop & Tra & $\begin{array}{c}\% \\
\text { Tra }\end{array}$ & $\mathrm{N}$ & $\%$ & $\mathrm{~N}$ & $\%$ & $\mathrm{~N}$ & $\%$ & $\mathrm{~N}$ & $\%$ & $\mathrm{~N}$ & $\%$ & $\mathrm{~N}$ & $\%$ & $\mathrm{~N}$ & $\%$ \\
\hline 1 & 22 & 14 & 63.6 & & & & & 2 & 14.2 & 1 & 7.1 & & & 11 & 78.5 & & \\
\hline 2 & 43 & 16 & 37.2 & 2 & 12.5 & 2 & 12.5 & 4 & 25.0 & , & & 1 & 6.25 & 7 & 43.7 & & \\
\hline 3 & 26 & 11 & 42.3 & & & & & & & & & & & 11 & 100 & & \\
\hline 4 & 28 & 13 & 46.2 & & & & & & & 3 & 23.0 & & & 10 & 76.9 & & \\
\hline 5 & 29 & 13 & 44.8 & 1 & 7.6 & & & 1 & 7.6 & & & 2 & 15.3 & 9 & 69.2 & 3 & 23.0 \\
\hline Total & 148 & 67 & 47.2 & 3 & 4.2 & 2 & 2.8 & 7 & 10.0 & 4 & 5.7 & 3 & 4.2 & 48 & 68.5 & 3 & 4.28 \\
\hline
\end{tabular}

*PREST + V1E : présent + verbe à 1 état *PREST + V1E + «-goiss » : présent + verbe à 1 état + « goiss »12 *PREST + V2E : présent + verbe à 2 état *PREST + V2E + «-goiss » : présent + verbe à 2 état + +-goiss»

*P1 (passé 1) +V1E : V1E +ass (^Ss)-da *P1+ V2E : (passé 1) + V2E : V2E + ass(^ss) -da *Gérondif : le morphème '-daga' '- $m^{\wedge} n s^{\wedge}$ ' équivalent au gérondif en français. 
Tableau 11: Distribution des adverbes temporels et des connecteurs dans les propositions de la TRAME des récits en coréen

\begin{tabular}{|c|c|c|c|c|c|c|c|c|}
\hline & & & $\begin{array}{l}\text { Conn } \\
(\mathrm{CT})^{*}\end{array}$ & & cempor & & $\begin{array}{l}\text { connecteurs } \\
(\mathrm{CO})^{*}\end{array}$ & $\begin{array}{ll}\text { adverbes } & \text { temporels } \\
(\mathrm{ADV})^{*} & \end{array}$ \\
\hline Enfant & Tprop & Tra & $\begin{array}{l}\text { (et) } \\
\text { puis }\end{array}$ & $\begin{array}{l}\text { (et) } \\
\text { après }\end{array}$ & $\begin{array}{l}\text { (et) } \\
\text { alors }\end{array}$ & et & & \\
\hline 1 & 22 & 14 & & 5 & 1 & 1 & & \\
\hline 2 & 43 & 16 & & & 2 & & mais (2) & juste après(1) \\
\hline 3 & 26 & 11 & & & 3 & 1 & mais (2) & pd ce $t^{\circ}-l a ̀(1)$ \\
\hline 4 & 28 & 13 & & & & 1 & mais (2) & \\
\hline 5 & 29 & 13 & & & 3 & 1 & $\begin{array}{l}\text { mais (1) comme } \\
\text { (1) }\end{array}$ & \\
\hline Total & 148 & 67 & & 5 & 9 & 4 & $\begin{array}{l}\text { mais (7), comme } \\
\text { (1) }\end{array}$ & $\begin{array}{l}\text { pd ce } t^{\circ} \text {-là(1) juste } \\
\text { après(1) }\end{array}$ \\
\hline
\end{tabular}

${ }^{*} \mathrm{CO}: \mathrm{s}^{\wedge}$ (comme/ car / parce que), $\mathrm{g}\left(\left.\right|^{\wedge}\right.$ nde (mais)

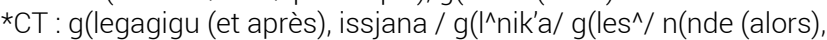

*ADV temporels : g(dongan (pendant ce temps-là) , ja (juste après que)

Dans les phrases complexes enchaînées par des suffixes verbaux conjonctifs, le temps du dernier verbe de la phrase gouverne celui des phrases précédentes. Regardons cet emploi avec des énoncés tirés de l'histoire du CHAT racontée en coréen par un des enfants bilingues que nous allons analyser :

\begin{tabular}{|l|}
\hline Enf4: \\
\hline 5. musîn/musîn goyangi-ga wagagi -go \\
\hline quoi/quoi chat -NOM venir -CONJ \\
\hline (quoi/quoi le chat est arrive) \\
\hline 6. scki -lîl bo -gu -yo \\
\hline les petits -ACC voir -CONJ-HON \\
\hline (et (le chat) a regardé les petits.) \\
\hline 7. m^gîmjig /m^g -gusip^ -gagigu \\
\hline délicieux /manger -avoir.envie.de-CONJcaus \\
\hline
\end{tabular}


(comme (le chat) avait envie de manger les petits qui avaient l'air délicieux)

8. $m^{\wedge}$ gîl -lagîl -ass $\quad-^{\wedge} \quad-$ yo

manger -BUT -PAST -DEC-HON

((il) est allé pour manger.) gouvernées par le temps de l'énoncé 8 , car ce sont des propositions à suffixe verbal conjonctif. Les occurrences de propositions dépendantes à la temporalité régie ont été compté comme des occurrences du temps régissant de la derrière proposition.

Les tableaux 10 et 11 donnent la répartition des marques linguistiques de la temporalité dans les propositions de la trame des récits en coréen. Il faut d'abord remarquer que les récits en coréen sont tous racontés sur la base de la séquentialité chronologique et/ou causative marquée par des suffixes verbaux conjonctifs.

Le nombre total des propositions (147 prop. en français vs 148 prop. en coréen) et le pourcentage de la trame ( $46.9 \%$ en français vs $47.2 \%$ en coréen) sont voisins dans les deux langues. Pour construire la trame du récit en coréen, les enfants utilisent à $68.5 \%$ la forme verbale passé 1 + verbe à 2 états du type 'casser', 'sauter' 'démolir' et aussi le présent + verbe à 2 états. De même que dans les récits en français, les connecteurs temporels peuvent précéder la proposition portant la morphologie verbale indiquée ci-dessus.

Nous pouvons dire qu'en coréen, les connecteurs temporels sont sémantiquement plus lourds, car ce n'est pas la temporalité qui prime, mais la relation de cause à effet, alors qu'en français, ce sont les relations purement temporelles, l'ordre chronologique, qui primeront sur la relation de cause à effet, car il n'y a pas de moyen naturel simple dans le récit de présenter l'ordre chronologique en spécifiant une relation causale. Le seul connecteur qui soit utilisé pour marquer explicitement la trame est 'et après', 'juste après' et le connecteur non temporel 'mais'.

Tableau 12 : Distribution des marques morphologiques dans les propositions de l'ARRIERE-PLAN des récits en coréen

\begin{tabular}{|c|c|c|c|c|c|c|c|c|c|c|c|c|c|c|c|c|c|c|c|}
\hline & & & & & $\begin{array}{l}\text { PREST } \\
+\mathrm{V} 1 \mathrm{E}\end{array}$ & & $\begin{array}{l}\text { PREST } \\
+\mathrm{V} 1 \mathrm{E} \\
\text { «goiss» }\end{array}$ & & $\begin{array}{l}\text { PREST } \\
+\mathrm{V} 2 \mathrm{E}\end{array}$ & & $\begin{array}{l}\mathrm{P} 1 \\
+\mathrm{V} 1 \mathrm{E} » \\
\text { «goiss» }\end{array}$ & & $\begin{array}{l}\text { P1 } \\
+ \text { V1E }\end{array}$ & & $\begin{array}{l}\mathrm{P} 1 \\
+\mathrm{V} 2 \mathrm{E} \ll\end{array}$ & & $\begin{array}{l}\mathrm{P} 1 \\
+\mathrm{V} 2 \mathrm{E} \\
\text { «goiss» }\end{array}$ & & G \\
\hline Enf & $\begin{array}{l}\mathrm{T} \\
\text { Pror }\end{array}$ & AP & $\begin{array}{c}\% \\
\text { AP }\end{array}$ & $\begin{array}{l}\mathrm{N} \\
0\end{array}$ & $\%$ & $\mathrm{~N}$ & $\%$ & ${ }_{0}^{N}$ & $\%$ & ${ }_{0}^{N}$ & $\%$ & ${ }_{0}^{N}$ & $\%$ & $\begin{array}{l}\mathrm{N} \\
0\end{array}$ & $\%$ & $\begin{array}{l}\mathrm{N} \\
0\end{array}$ & $\%$ & $\mathrm{~N}$ & $\%$ \\
\hline 1 & 22 & 8 & 36.3 & & & & & & & 1 & 12.5 & 3 & 37.5 & 2 & 25 & & & 1 & 12.5 \\
\hline 2 & 43 & 27 & 62.7 & 11 & 40.7 & 1 & 3.7 & 1 & 3.7 & 1 & 3.7 & 1 & 3.7 & 5 & 18.5 & & & & \\
\hline 3 & 26 & 15 & 57.6 & 2 & 13.3 & 1 & 6.6 & 1 & 6.6 & 2 & 13.3 & 2 & 13.3 & 2 & 13.3 & 1 & 6.6 & 2 & 13.3 \\
\hline 4 & 28 & 14 & 51.8 & & & 1 & 7.1 & 2 & 14.2 & & & 7 & 50.0 & 2 & 14.2 & & & 2 & 14.2 \\
\hline
\end{tabular}




\begin{tabular}{|l|l|l|l|l|l|l|l|l|l|l|l|l|l|l|l|l|l|}
\hline 5 & 29 & 14 & 44.8 & & & 2 & 12.5 & & & 1 & 6.25 & 5 & 31.2 & 3 & 18.7 & \\
\hline \hline Total & 148 & 78 & 52.7 & 13 & 16.6 & 5 & 6.4 & 4 & 5.1 & 5 & 6.4 & 18 & 23.0 & 14 & 17.9 & 1 & 1.2 \\
\hline
\end{tabular}

* enf : enfant

Tableau 13 : Distribution des adverbes temporels et des connecteurs dans les propositions de I'ARRIERE-PLAN des récits en coréen

\begin{tabular}{|c|c|c|c|c|c|c|c|c|}
\hline & & & Connecte & eurs & tempor & & connecteurs & adverbes temporels \\
\hline Enf. & TProp & AP & (et)alors & et & $\begin{array}{l}\mathrm{r} \text { en } \\
\text { ar* }^{*}\end{array}$ & $\mathrm{PR}^{*}$ & & \\
\hline 1 & 22 & 8 & 1 & 2 & 1 & 1 & mais(1)comme(1) & toujours(1) \\
\hline 2 & 43 & 27 & & & 2 & & $\begin{array}{l}\text { mais(2)car(1)comme } \\
\text { (3)parce que(1) }\end{array}$ & \\
\hline 3 & 26 & 15 & 1 & 1 & 2 & 2 & & $\begin{array}{l}\text { un jour(1)au } \\
\text { moment où(1) }\end{array}$ \\
\hline 4 & 28 & 14 & 1 & 2 & 4 & 2 & mais(1)comme(1) & \\
\hline 5 & 29 & 14 & 1 & & 2 & & mais(2) comme(1) & \\
\hline Total & 148 & 78 & 4 & 5 & 11 & 5 & $\begin{array}{l}\text { mais(5)comme(6) } \\
\operatorname{car}(1) p q(1)\end{array}$ & $\begin{array}{l}\operatorname{tjrs}(1) \text { un jour(1)au } \\
m^{\circ} \text { où(1) }\end{array}$ \\
\hline
\end{tabular}

$\star_{r}$ en ar : retours en arrière * $P R G$ : progressif *ADV : g(dongan (pendant ce temps-là)

44 Les marques morphologiques utilisés dans les propositions de l'AP sont le morphème temporel $\mathrm{P} 1+\mathrm{V}$ à 1 état (soit $23 \%$ ) et $\mathrm{PREST}+\mathrm{V}$ à 1 état (soit $16.6 \%$ ). Nous trouvons la forme $\mathrm{P} 1+\mathrm{V}$ à 2 états toute seule. Cette forme est employée dans le cas de relation de cause, le moment de la situation ultérieure formant ainsi le cadre de l'événement. Quant à l'enfant 2 qui, de même que pour le récit français, raconte son récit en utilisant le présent de narration, elle utilise la morphologie verbale du present $+V$ à 0 état ou 1 état, ou la morphologie du présent $+\mathrm{V}$ à 2 états+ "goiss ", ainsi que les pronoms anaphoriques 'ça' ou 'cet événement' qui réfèrent explicitement à un événement précédent. Nous trouvons ainsi les adverbes temporels de type 'pendant ce temps-là', 'au moment où', 'quand', le gérondif et aussi le retour en arrière avec la même fonction que pour les autres enfants. Nous pouvons conclure que pour les propositions de l'AP, les enfants utilisent en coréen les moyens suivants : le morphème temporel du passé $1+$ verbe à 0 ou 1 état ou bien le passé 1 + verbe à 1 états + " goiss » et le morphème temporel du présent+ verbe à 0 ou 1 état ou bien le présent + verbe à 1 états + "goiss ». Il y a donc une plus grande variété de moyens que dans la trame.

On relève aussi d'autres moyens discursifs tels que la configuration cadre duratif événement inclus : une situation durative est la cause d'une deuxième situation pouvant être ou non durative (procès à 1 état ou 2 états inclus dans procès à 1 état). 


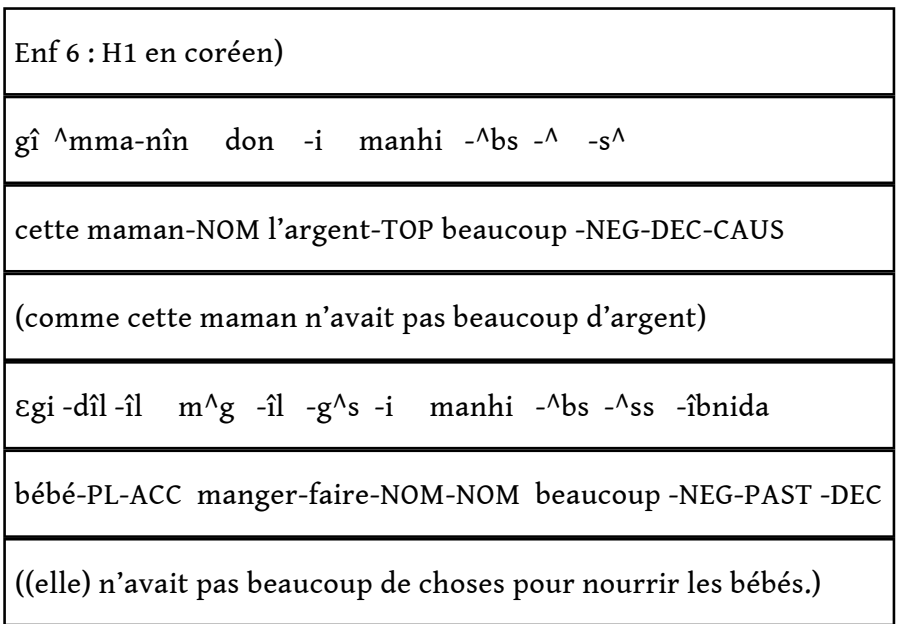

Notamment les propositions avec le connecteur causal '- $\mathrm{s}^{\wedge}$ ' (='comme', 'car', 'puisque' et 'parce que') dans les récits en coréen permettent de les caractériser comme des récits fondés sur la causalité présentée en ordre chronologique.

La comparaison entre les récits en français et ceux en coréen nous inspire les réflexions suivantes :

A partir des résultats des analyses et des observations faites sur l'utilisation des structures temporelles du français chez les enfants coréens, et des analyses similaires sur leurs récits en coréen, on peut se rendre compte que les enfants utilisent la morphologie verbale dans les deux langues d'une manière assez similaire.

Nous trouvons également dans les récits des deux langues une distribution très systématique entre les plans du discours trame vs arrière-plan des traits temporels inhérents aux situations: dans les propositions d'arrière-plan on trouve des procès duratifs et sans clôture inhérente, et dans celles de la trame, des procès téliques, à deux états, en français comme en coréen. On peut en inférer que la structure narrative canonique fournit un appui important aux enfants pour la mise en œuvre des moyens linguistiques de la temporalité dans le récit.

\section{Conclusion}

50 A travers ces analyses, nous avons observé que la distribution de la morphologie verbale dans les deux langues est assez similaire et qu'elle est liée à la structuration globale du récit, c'est-à-dire à l'opposition T/AP. Cela nous permet de dire que les enfants bilingues coréens s'appuient sur la distinction sémantique entre types de procès et sur l'organisation macro-structurelle T/AP pour formuler le temps et l'aspect dans les deux langues : ils réservent de préférence la trame à des événements à deux états et l'arrièreplan à des situations à 1 ou 0 état, et ils utilisent surtout des formes verbales à valeur perfective dans la trame, et imperfective dans l'arrière-plan. Il faudrait comparer ce cas d'acquisition à des données issues d'enfants du même âge monolingues locuteurs natifs du français d'une part, et du coréen d'autre part, afin de pouvoir interpréter pour ce qui est de notre situation d'acquisition ce processus d'appui sur le sémantisme des verbes dans la construction du temps et de l'aspect. 
D'après le travail de Sénémaud (1997), les enfants français monolingues des mêmes âges, dans les mêmes types d'histoires sur images, ont un pourcentage de trame plus élevé ( $65 \%$, en moyenne) que les enfants bilingues étudiés ici ( $40 \%$, en moyenne), et utilisent plus de connecteurs de successivité et d'organisateurs temporels que nos enfants. Mais à la différence des enfants bilingues franco-coréens, ils utilisent très peu de connecteurs causaux ${ }^{13}$.

On peut constater aussi que les récits en coréen sont fortement appuyés sur des relations de cause à effet, alors qu'en français ce sont les relations purement temporelles entre événements, l'ordre chronologique, qui priment.

Pour tenter d'expliquer nos constatations, on peut examiner les caractéristiques typologiques pertinentes du coréen vs du français. Il faudra regarder plus de données pour généraliser cet aspect, mais on peut avancer que la macro-structure du récit en coréen se fonde davantage sur des chaînes causales entre événements causateurs événements conséquences que les récits en français, qui contiennent très peu de relations causales (ces tendances sont claires au moins pour ce type de récits sur images).

Cet article synthétise une première étape de notre recherche en cours sur l'acquisition de la temporalité chez des enfants bilingues pour un couple de langues typologiquement très éloignées. Ses résultats nous permettent maintenant d'aborder une autre étape ciblée sur la conceptualisation et la formulation des différentes relations temporelles entre événements, ainsi que sur leurs moyens d'expression dans la construction d'énoncés complexes (parataxe / hypotaxe) dans le récit.

\section{BIBLIOGRAPHIE}

ADAM, J.M (1984) : Le récit, Paris, QSJ.

CHOI, J.N. (1996) : La temporalité dans les récits sur images d'enfants bilingues franco-coréens dans leurs deux langues. Mémoire de D.E.A. de Sciences du langage, Université de Paris XNanterre.

FAYOL, M (1985) : Le récit et sa construction, Paris. Delachaux \& Niestlé.

FRANÇOIS, F (1984) : Conduites linguistiques chez le jeune enfant. Paris, PUF.

GROSJEAN, F (1982): Life with two languages. An introduction to bilingualism. London, Harvard University Press.

HICKMANN, M. \& LIANG, J. (1990): “ Clause-structure variation in Chinese narrative discourse: a developmental analysis. " Linguistics 6, pp. 1167-1200.

HICKMANN, M., LIANG, J., HENDRIKS, H., \& ROLAND, F., (1990): The development of discourse cohesion. A coding Manual. Nijmegen: Max Planck Institute for Psycholinguistics.

KIM C.K.(1990): ulimal sijewa sang( $\mathrm{y}^{\wedge}$ ngu (L'étude des temps et aspects du coréen) Séoul, Tehagsa.

KLEIN, W (1989) : L’Acquisition de langue étrangère, Paris. Armand Colin. 
KLEIN, W. \& VON STUTTERHEIM C. (1989) : “ Referential mouvement in descriptive and narrative discourse. " In R. Dietrich \& C. Graumann, (eds) : Language processing in social context. Amsterdam, Elsevier, pp. 39-76.

KLEIN, W. \& VON STUTTERHEIM C. (1991) : « Text structure and referential mouvement »-Sprache und Pragmatik, N. 22, pp. 1-32.

KLEIN, W (1995): Time in language. London, Routledge.

LEVELT, W. (1989): Speaking. From Intention to Articulation. Cambridge, Massachussets, M.I.T Press.

NOYAU, C. (1988) : «Conter, redire : cada vez es otro cuento. »In : Actes du Troisième Colloque de Linguistique Hispanique. Grenoble, Université Stendhal, pp. 26-56.

NOYAU, C. (1990) : "Structure conceptuelle, mise en texte et acquisition d'une langue étrangère." Langages 100,pp. 101-114.

NOYAU, C. (1991) : Le temps dans le discours. Construction du récit. Thèse d'habilitation. Université de Paris VIII. vol. 1.

SCHLYTER, S. (1990): « The acquisition of tense and aspect » In: J Meisel, ed. Two first languages. Early Grammatical Development in Bilingual Children. Dordrecht, Foris.

SCHLYTER, S. (1995) : « Formes verbales du passé dans des interactions en langue forte et en langue faible » - AILE (Acquisition et Interaction en Langue Etrangère), № 6, pp. 129-152.

SENEMAUD, M. (1997) : L'enfant et le récit : étude sur la temporalité dans des récits d'enfants en vue d'une recherche sur les récits d'enfants dysphasiques. Mémoire de maîtrise, Université Paris X-Nanterre.

\section{ANNEXES}

\section{Abréviations utilisées}

\section{Coréen :}

\begin{tabular}{|l|l|l|l|}
\hline $\mathbf{N}:$ & particule nominale & V : & particule verbale \\
\hline ACC & $:$ particule d'accusatif (N) & CAUS & $:$ particule causative (V) \\
\hline CIT & $:$ particule citative (V) & COM & $:$ particule de comitatif (N) \\
\hline COMPAR & $:$ particule de comparatif & CONJ & $:$ particule de conjonction \\
\hline CONC & $:$ particule concessive (V) & DAT & $:$ particule de datif (N) \\
\hline DEC & $:$ particule déclarative (V) & DIR & $:$ particule de direction (N) \\
\hline \hline EXCLA & $:$ particule exclamative (V) & EXHOR & $:$ particule exhortative (V) \\
\hline \hline FUT & $:$ particule du temps au futur (V) & GEN & $:$ particule de génitif (N) \\
\hline
\end{tabular}




\begin{tabular}{|l|l|l|l|}
\hline HON & $:$ particule honorifique $(\mathrm{V})$ & IMP & : particule impérative $(\mathrm{V})$ \\
\hline INSTR & $:$ particule d'instrumental (N) & INT & $:$ particule interrogative $(\mathrm{V})$ \\
\hline LOC & $:$ particule de locatif (N) & NEG & $:$ particule négative $(\mathrm{V})$ \\
\hline NOM & $:$ particule de nominatif (N) & PAST & $:$ particule de temps passé (V) \\
\hline PL & $:$ particule de pluriel (N) & PREST & $:$ particule de temps présent (V) \\
\hline SPEC & $:$ spécifieur & TOP & $:$ particule de topique (N) \\
\hline Autres : & & & \\
\hline T & $:$ trame & AP : arrière-plan & \\
\hline
\end{tabular}

\section{NOTES}

1. Cet article a pour origine un travail effectué pour un mémoire de D.E.A. intitulé 'La temporalité dans les récits sur images d'enfants bilingues franco-coréens dans leurs deux langues.' (Paris X-Nanterre, octobre 1996).

2. Dans le cadre de la recherche pour la thèse intitulée 'Conceptualisation et formulation linguistique de la temporalité et des entités dans les récits d'enfants bilingues franco-coréens dans leurs deux langues', nous examinons la relation entre la conceptualisation et la formulation de représentations d'événements complexes, et la trajectoire développementale des moyens linguistiques pour la référenciation au temps et aux entités chez les enfants, à travers 120 récits d'enfants bilingues français-coréens en situation de bilinguisme consécutif.

3. Pour ces enfants, même pour ceux qui sont nés en France, l'accès à la L2 commence à l'âge de 3 ou 4 ans à l'école maternelle. Pour la caractérisation des différentes stratégies d'acquisition de deux langues voir F. Grosjean (1982) : Life with two languages, p.180.

4. Pour définir la langue forte et la langue faible, je m'appuie d'abord sur l'entretien que j'ai fait avant l'enregistrement des récits sur l'attitude et le degré d'aisance vis-à-vis du français et du coréen et par ailleurs, parmi les critères de S. Schlyter (1994: 69), j'ai retenu essentiellement le choix de la langue dans des contextes bilingues.

5. Ce type de question à laquelle le locuteur répond en produisant des textes cohérents est l'aspect central du modèle de la Quaestio par Klein et Von Stutterheim $(1989,1991)$. Nous détaillons ce modèle plus loin.

6. Hei-in, qui était la plus grande et la plus compétente en français parmi les enfants réunis pour la soirée, a considéré la tâche comme un jeu, et a déclenché une ambiance agréable pour que les enfants se mettent à parler en français. Il faut noter que faire parler les enfants coréens en français entre eux en milieu coréen n'est pas évident.

7. Certaines formes verbales sont transcrites phonétiquement pour ne pas préjuger de l'interprétation linguistique.

8. On trouve 4 notions aspectuelles, perfectif, imperfectif, parfait et prospectif : quand je suis rentré, il était sous la douche. MQ IN (intérieur à) MS : imperfectif pendant qu'il était sous la douche, le téléphone a sonné. MQ INCL (inclut) MS : perfectif ça y est, elle a cassé une assiette MQ APR (après) MS : parfait tiens, il va pleuvoir MQ AV (avant) MS : prospectif . Pour plus de détail, voir Noyau (1991). 
9. La différence entre le nombre total de propositions des différents types et le total des colonnes par morphèmes temporels est due aux propositions qui n'ont pas de localisation temporelle, par exemple les infinitifs (ex. enfant 1 : le nombre total de propositions de la Trame est de 12 propositions, mais le nombre total des colonnes par morphèmes temporels est de 11 propositions.)

10. Sénémaud, M (1997) : L'enfant et le récit : étude sur la temporalité dans des récits d'enfants en vue d'une recherche sur les récits d'enfants dysphasiques. Mémoire de maîtrise Université Paris XNanterre.

11. Enf 5 indique le numéro d'ordre de chaque enfant (enfant 5). $\mathrm{H}$ indique l'histoire, 1 (l'histoire du CHAT) et 2 (l'histoire du CHEVAL).

12. «-goiss »: morphème progressif. «-goiss» est l'équivalent du marqueur progressif périphrastique du français 'être en train de'.

13. Voir le Tableau 25 de Sénémaud 1997 (73).

\section{RÉSUMÉS}

Nous nous intéressons à l'acquisition bilingue successive enfantine chez des enfants coréens habitant en France. La présente étude porte sur 20 récits à partir d'images enregistrés auprès de 5 enfants bilingues dans leurs deux langues (enfants de 7 à 9 ans, résidant en France depuis plus de 3 ans, et scolarisés depuis 14 mois à 4 ans 1/2). A travers l'analyse de ces productions, nous étudions quelques aspects centraux de la construction du récit : 1) la macro-structure des récits, notamment la répartition des propositions entre la trame et l'arrière-plan, et la façon dont l'enfant sélectionne et met en relation les différents procès constituant un macro-événement ; 2) la micro-structure pour ce qui est de la temporalité : sélection des procès, temporalité inhérente de ceux-ci ; relations temporelles, marquage morphologique de la temporalité, de l'aspectualité, et connecteurs.

On observe : a) que la distribution de la morphologie verbale dans les deux langues est assez similaire et qu'elle est liée à la structuration globale du récit, c'est-à-dire à l'opposition trame / arrière-plan; b) que les récits en coréen sont fortement appuyés sur des relations de cause à effet, alors qu'en français ce sont les relations purement temporelles entre événements qui priment. Nous en concluons que ces enfants bilingues franco-coréens s'appuient sur la distinction sémantique entre types de procès et sur l'organisation macro-structurelle trame/ arrière-plan pour formuler le temps et l'aspect dans leurs deux langues: ils réservent de préférence la trame à des événements transitionnels et l'arrière-plan à des situations statiques, et ils utilisent surtout des formes verbales à valeur perfective dans la trame, et imperfective dans l'arrière-plan.

This study focuses on the successive bilingual language acquisition of Korean children who live in France. This study analyses 20 picture-based narratives elicited from 5 bilingual children in both languages ( 7 - 8 years old, living in France for more than 3 years). The study is based on central aspects of linguistic construction of narrative discourse: 1) macro-structure of narrative discourse : distribution of clauses into foreground and background, selection of the events that constitute the macro-event and relations between them;2) micro-structure as regards temporality: choice of events, temporal characteristics of the events; temporal relations, morphological tense and aspect, and connectors. 
We observe that : a) the distribution of verb morphology in the two languages is very similar, and this tendency is linked to the global structure of the text, i.e. the foreground/background distinction ; b) the Korean narratives are organized around a cause/effect relationship, whereas in French, they stick to chronological order.

We conclude that these bilingual children rely on the semantic distinction between types of events and on the organization of the global structure foreground / background to express tense and aspect in the two languages: foreground propositions mainly contain 2-state events and background propositions 0-state events (properties) or 1-state events (states), and they tend to use perfective verbal forms in the foreground, and imperfective in the background.

\section{AUTEUR}

JIN-NAM CHOI

Université de Paris X-Nanterre, EA 372 Modèles Linguistiques et Dynamique des Langues et Groupement de Recherche 113 du CNRS, jnchoi@club-internet.fr 